\title{
Multi-mycotoxin analysis using dried blood spots and dried serum spots
}

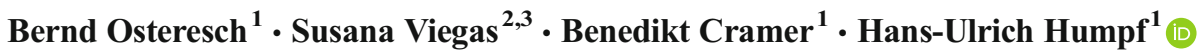

Received: 9 December 2016 / Revised: 8 February 2017 / Accepted: 24 February 2017 / Published online: 15 March 2017

(C) The Author(s) 2017. This article is published with open access at Springerlink.com

\begin{abstract}
In this study, a rapid multi-mycotoxin approach was developed for biomonitoring and quantification of 27 important mycotoxins and mycotoxin metabolites in human blood samples. HPLC-MS/MS detection was used for the analysis of dried serum spots (DSS) and dried blood spots (DBS). Detection of aflatoxins $\left(\mathrm{AFB}_{1}, \mathrm{AFB}_{2}, \mathrm{AFG}_{1}, \mathrm{AFG}_{2}, \mathrm{AFM}_{1}\right)$, trichothecenes (deoxynivalenol, DON; DON-3-glucoronic acid, DON-3-GlcA; T-2; HT-2; and HT-2-4-GlcA), fumonisin $\mathrm{B}_{1}\left(\mathrm{FB}_{1}\right)$, ochratoxins (OTA and its thermal degradation product 2'R-OTA; OT $\alpha$; 10hydroxychratoxin A, 10-OH-OTA), citrinin (CIT and its urinary metabolite dihydrocitrinone, DH-CIT), zearalenone and zearalanone (ZEN, ZAN), altenuene (ALT), alternariols (AOH; alternariol monomethyl ether, AME), enniatins (EnA, EnA ${ }_{1}$, $\mathrm{EnB}, \mathrm{EnB}_{1}$ ) and beauvericin (Bea) was validated for two matrices, serum (DSS), and whole blood (DBS). HPLC-MS/MS analysis showed signal suppression as well as signal enhancement due to matrix effects. However, for most analytes LOQs in the lower $\mathrm{pg} / \mathrm{mL}$ range and excellent recovery rate were achieved using matrix-matched calibration. Besides validation of the method, the analyte stability in DBS and DSS was also investigated.
\end{abstract}

Electronic supplementary material The online version of this article (doi:10.1007/s00216-017-0279-9) contains supplementary material, which is available to authorized users.

Hans-Ulrich Humpf

humpf@wwu.de

Institute of Food Chemistry, Westfälische Wilhelms-Universität Münster, Corrensstr. 45, 48149 Münster, Germany

2 Environment and Health RG, Lisbon School of Health Technology, Polytechnic Institute of Lisbon, Av. D. João II, Lote 4.69.01, Parque das Nações, 1990-096 Lisbon, Portugal

3 Centro de Investigação em Saúde Pública, Escola Nacional de Saúde Pública, Universidade NOVA de Lisboa, Avenida Padre Cruz, 1600-560 Lisbon, Portugal
Stability is a main issue for some analytes when the dried samples are stored under common conditions at room temperature. Nevertheless, the developed method was applied to DBS samples of a German cohort $(n=50)$. Besides positive findings of OTA and 2'R-OTA, all samples were positive for EnB. This methodical study establishes a validated multi-mycotoxin approach for the detection of 27 mycotoxins and metabolites in dried blood/serum spots based on a fast sample preparation followed by sensitive HPLC-MS/MS analysis.

Keywords Biomonitoring · Dried blood spot $\cdot$ Dried serum spot $\cdot$ HPLC-MS/MS · Mass spectrometry $\cdot$ Mycotoxin

$\begin{array}{ll}\text { Abbreviations } & \\ \text { 2'R-OTA } & \text { 2'R-Ochratoxin A } \\ \text { 10-OH-OTA } & \text { 10-Hydroxyochratoxin A } \\ \text { AFB }_{1} & \text { Aflatoxin } B_{1} \\ \text { AFB }_{2} & \text { Aflatoxin } B_{2} \\ \text { AFG }_{1} & \text { Aflatoxin } \mathrm{G}_{1} \\ \text { AFG }_{2} & \text { Aflatoxin } \mathrm{G}_{2} \\ \text { AFM }_{1} & \text { Aflatoxin } \mathrm{M}_{1} \\ \text { ALT }_{\text {AME }} & \text { Altenuene } \\ \text { AOH } & \text { Alternariol monomethyl ether } \\ \text { BEA }_{\text {DBS }} & \text { Alternariol } \\ \text { DH-CIT } & \text { Deauvericin } \\ \text { DON } & \text { Dihydrocitrinone } \\ \text { DON-3-GlcA } & \text { Deoxynivalenol } \\ \text { DSS } & \text { DON-3-glucuronide } \\ \text { EnA } & \text { Enniatin A serum spot } \\ \text { EnA } & \text { Enniatin A } \\ \text { EnB } & \text { Enniatin B } \\ \text { EnB } & \text { Enniatin B } \\ & \end{array}$




$\begin{array}{ll}\text { FB }_{1} & \text { Fumonisin } B_{1} \\ \text { HT-2 } & \text { HT-2 toxin } \\ \text { HT-2-4-GlcA } & \text { HT-2-toxin-4-glucuronide } \\ \text { OTA } & \text { Ochratoxin A } \\ \text { OT } \alpha & \text { Ochratoxin } \alpha \\ \text { sMRM } & \text { Scheduled multiple reaction monitoring } \\ \text { T-2 } & \text { T-2 toxin } \\ \text { ZAN } & \text { Zearalanone } \\ \text { ZEN } & \text { Zearalenone }\end{array}$

\section{Introduction}

Mycotoxins are toxic secondary metabolites produced by molds contaminating food during production, shipping, processing, or storage [1]. In order to protect consumers' health maximum levels for food and feed have been set by regulatory authorities [2]. Regulatory attempts are usually based on food contamination and consumption data. However, this approach does not take individual exposure due to personal, regional as well as cultural divergences into account [3]. Biomarker-based approaches are more and more used to assess dietary exposure, as they allow to analyze physiological samples like urine or blood for each test person individually [4]. Various sample preparation and quantification techniques are described for mycotoxins and their metabolites in several physiological samples [5-9]. Main challenges in biomonitoring-based methods are usually low analyte concentrations and matrix interferences during analysis. Recently developed dilute-and-shoot approaches for urine attempt to remove the majority of matrix compounds by chromatographic separation and take advantage of highly sensitive mass spectrometers [10,11].

For blood analysis, dried blood spots (DBS) currently undergo a comeback concerning medical or forensic issues mainly due to improved detection limits and therefore leading to new fields of application [12,13]. For example, DBS are suitable for extensive biomonitoring studies of environmental contaminants in humans or animals [14]. Particularly, for animal studies the application of DBS is an effective improvement concerning sample collection as only limited blood volumes are often available for analysis due to the low body weight of small animals [15]. Advantages of DBS compared to conventional blood collection are the minimally invasive sampling, simple sample preparation, easier storage, and shipping as well as the small volume required [16]. Samples can be collected on filter paper cards by heel, ear, or finger pricking as well as by spotting a known blood volume from ampoules after conventional vain puncture [17, 18]. Even if DBS are the standardized basis for medical tests, dried serum spots (DSS) are an additional opportunity for immunological tests and other blood counts. Likewise to DBS, DSS take advantage of simplified storage and shipment conditions $[19,20]$.
Recently, a DBS method for the detection of ochratoxin A in dried blood spots using HPLC-MS/MS has been published including the optimization of various basic parameters such as spotting volume and hematocrit which did not have a strong influence $[21,22]$. Thus, the application of DBS for mycotoxin analysis showed positive findings in all samples for OTA as well as $2^{\prime} R$-OTA in the blood of coffee drinkers [21]. Here, we present the further development of this method by the incorporation of 27 relevant mycotoxins and metabolites and its application to serum and blood samples. Compounds monitored were aflatoxins $\left(\mathrm{AFB}_{1}, \mathrm{AFB}_{2}, \mathrm{AFG}_{1}, \mathrm{AFG}_{2}, \mathrm{AFM}_{1}\right)$, Alternaria toxins (ALT, AME, AOH), citrinin (CIT), and its metabolite dihydrocitrinone (DH-CIT). Furthermore, trichothecenes as deoxynivalenol (DON), deoxynivalenol-3-glucuronide (DON3-GlcA), T-2-toxin (T-2), HT-2-toxin (HT-2), HT-2-toxin4-glucoronide (HT-2-4-GlcA) have been incorporated. In addition, the structurally related cyclic hexadepsipeptides beauvericin (BEA) and enniatins $\left(\mathrm{EnA}, \mathrm{EnA}_{1}, \mathrm{EnB}, \mathrm{EnB}_{1}\right)$ are included. Lastly, fumonisin $\mathrm{B}_{1}\left(\mathrm{FB}_{1}\right)$, ochratoxin $\mathrm{A}$, and its thermal degradation product $2^{\prime} R$-ochratoxin $\mathrm{A}$ as well as ochratoxin $\alpha$ and 10-hydroxyochratoxin A (OTA, 2'R-OTA, OT $\alpha, 10$-HOTA), zearalenone (ZEN), and zearalanone (ZAN) are enclosed [1, 23-25]. Those mycotoxins and metabolites are frequently determined in human fluids such as the urine, human breast milk, and blood, or serum and plasma, respectively [26-29]. Their reliable analysis in a multi-mycotoxin approach for physiological samples is a challenging task but allows to determine the individual mycotoxin exposure of humans and animals.

\section{Experimental}

\section{Safety consideration}

Mycotoxins should be handled with care due to their toxic effects for humans. Blood samples underlie biological hazard in principle. It is mandatory to autoclave all contaminated lab material, e.g., ampoules and paper collection cards, which encountered blood or serum.

\section{Chemicals and reagents}

Acetonitrile (ACN) and acetone were of LC gradient purity (VWR, Darmstadt, Germany). Type 1 laboratory water was used (Resistivity $>18 \mathrm{M} \Omega, \mathrm{TOC}<10 \mathrm{ppb}$; Purelab flex, Veolia Water Technologies, Celle, Germany). Formic acid and acetic acid was purchased from Grüssing (Filsum, Germany) with $99.5 \%$ purity. Blood collection tubes were MonovetteR® EDTA KE/7.5 mL and Monovette ${ }^{\circledR}$ Serum-Gel from Sarstedt (Nümbrecht, Germany). Whatman 903 protein saver cards ${ }^{\mathrm{TM}}$ for sample collection and preparation were acquired from Sigma-Aldrich (Taufkirchen, Germany). 
$\mathrm{AFB}_{1 / 2}, \mathrm{AFG}_{1 / 2}, \mathrm{AFM}_{1}, \mathrm{BEA}, \mathrm{CIT}, \mathrm{EnA}, \mathrm{EnA}_{1}, \mathrm{EnB}$, $\mathrm{EnB}_{1}, \mathrm{FB}_{1}, \mathrm{FB}_{2}, \mathrm{ZAN}$, and $\alpha-/ \beta$-zearalenol were from Sigma-Aldrich. DH-CIT was purchased from AnalytiCon Discovery (Potsdam, Germany). DON, FB 1 , 10-OH-OTA, OTA, OT $\alpha$, T-2, HT-2, and ZEN were isolated and purified from fungal cultures [30-35]. 2' $R$-OTA was produced by thermal isomerization of OTA [25]. Glucuronic acid conjugates (DON-3-GlcA and HT-2-4-GlcA) were synthesized enzymatically using rat and pig liver microsomes [36]. ALT, AME, and $\mathrm{AOH}$ were isolated and purified according to a recently published procedure [37]. Tenuazonic acid was chemically synthesized by Lohrey et al. [38]. Stock solutions were prepared at concentrations of 10 or $20 \mu \mathrm{g} / \mathrm{mL}$ in ACN or ACN/ $\mathrm{H}_{2} \mathrm{O}$ and stored at $-20{ }^{\circ} \mathrm{C}$ in the dark until further use. Purity of in-house produced standards was checked by HPLC-DAD/ ELSD (evaporative light scattering detector) and was $\geq 98 \%$. Structure verification was done via MS and NMR. Aflatoxins $B_{1 / 2}$ and $G_{1 / 2}$ were applied as a combined solution at $2 \mu \mathrm{g} / \mathrm{mL}$ for $\mathrm{AFB}_{1} / \mathrm{AFG}_{1}$ and $0.5 \mu \mathrm{g} / \mathrm{mL}$ for $\mathrm{AFB}_{2} / \mathrm{AFG}_{2}$. Two working solutions were prepared at 20 -fold concentration of the highest calibration point at each day of measurement in $\mathrm{ACN} / \mathrm{H}_{2} \mathrm{O}(80: 20, v / v)$ leading to concentrations of $1 \mu \mathrm{g} / \mathrm{mL}$ for the majority of analytes. For $\mathrm{AFB}_{1} / \mathrm{AFG}_{1}$ the concentrations in the working solution were $200 \mathrm{ng} / \mathrm{mL}$ and for $\mathrm{AFB}_{2} /$ $\mathrm{AFG}_{2} 50 \mathrm{ng} / \mathrm{mL}$.

\section{Sample collection}

Human blood and serum samples were provided by healthy volunteers giving written consent as participants of biomonitoring studies in Germany (Ethical committee of the University Hospital Münster, Germany, file reference: 2014187 -f-S). Of each sample $100 \mu \mathrm{L}$ of blood was spotted four times on filter paper cards.

\section{Sample preparation}

Serum was prepared by whole blood collection in Monovette ${ }^{\circledR}$ Serum-Gel tubes and centrifugation at $3000 \times g$ for $10 \mathrm{~min}$. Afterwards, the supernatant was used as serum sample. For sample preparation, $100 \mu \mathrm{L}$ of whole blood or serum was pipetted on Whatman 903 protein saver cards ${ }^{\mathrm{TM}}$ and dried over night at room temperature to obtain DBS and DSS. In order to assure no overlapping of the DSS, only three of the five marked spotting positions were used when $100 \mu \mathrm{L}$ serum was applied to the cards, as serum spots show a larger diameter compared do DBS due to lower viscosity. Next, $100 \mu \mathrm{L}$ DBS or DSS were completely cut out leaving approx. $1 \mathrm{~mm}$ space between the cutting edge and the visually detected border of the spot, followed by extraction with $1 \mathrm{~mL}$ extraction mixture consisting of water/acetone/acetonitrile (30:35:35, v/v/v) in $2 \mathrm{~mL}$ safe-lock tubes for $30 \mathrm{~min}$ under sonication. After extraction, an aliquot of $800 \mu \mathrm{L}$ was transferred into a new 2-mL safe-lock tube and evaporated to dryness at $50^{\circ} \mathrm{C}$ under reduced pressure. The residues were reconstituted with water/acetonitrile/acetic acid (95:5:0.1, $v / v /$ v) followed by centrifugation at $22,000 \times \mathrm{g}$ for $10 \mathrm{~min}$ and injection of $30 \mu \mathrm{L}$ of the supernatant into the HPLC-MS/MS system. Sample preparation was performed in duplicate for the volunteer samples and in triplicate for each fortified recovery sample.

\section{HPLC-MS/MS setup}

Analysis was carried out on a 1260 Infinity LC system (Agilent, Waldbronn, Germany) coupled to a QTRAP 6500 mass spectrometer (SCIEX, Darmstadt, Germany). During method development four different column materials, Nucleodur $\mathrm{C}_{18}$ ISIS, Pyramid, Gravity, and Gravity SB (all Macherey-Nagel, Düren, Germany) were compared for retention behavior and peak shape (see Electronic supplementary material). Finally, a Nucleodur $\mathrm{C}_{18}$ Gravity SB column $(3 \mu \mathrm{m}, 2.0 \times 100 \mathrm{~mm})$ equipped with a guard column $(2.0 \times 4.0 \mathrm{~mm})$ of the same material was used at a column temperature of $45^{\circ} \mathrm{C}$. Eluent A was acetonitrile containing $2 \%$ acetic acid and eluent $\mathrm{B}$ was water with $0.1 \%$ acetic acid. The binary gradient and flow rate was set up as follows: $0 \mathrm{~min}, 3 \% \mathrm{~A}(750 \mu \mathrm{L} / \mathrm{min})$; $3 \mathrm{~min}, 15 \%$ A $(750 \mu \mathrm{L} / \mathrm{min}) ; 4.5 \mathrm{~min}, 55 \%$ A $(750 \mu \mathrm{L} /$ $\mathrm{min}) ; 6.0 \mathrm{~min}, 55 \% \mathrm{~A}(850 \mu \mathrm{L} / \mathrm{min}) ; 8.0 \mathrm{~min}, 100 \% \mathrm{~A}$ $(850 \mu \mathrm{L} / \mathrm{min}) ; 10.0 \mathrm{~min}, 100 \%$ A $(850 \mu \mathrm{L} / \mathrm{min})$; $10.1 \mathrm{~min}, 3 \%$ A $(750 \mu \mathrm{L} / \mathrm{min}) ; 11.5 \mathrm{~min}, 3 \%$ A $(750 \mu \mathrm{L} / \mathrm{min})$. Electrospray ionization (ESI) was performed in both polarities with +5500 or $-4500 \mathrm{~V}$ in scheduled multiple reaction monitoring ( $\left(\mathrm{SMRM}^{\mathrm{TM}}\right)$ detection mode. Latter increases the number of data points as less transitions are monitored at the same time. Furthermore, longer dwell times are possible resulting in decreased noise levels and better signal-to-noise ratios. Analyst 1.6.2 software was used for data acquisition. In order to avoid a high entry of unwanted polar compounds into the mass spectrometer, a diverter valve was applied between column and mass spectrometer allowing to discard the first $2.6 \mathrm{~min}$ of each chromatographic run before entering the ion source. ESI-source parameters were optimized and set to a source temperature of $500{ }^{\circ} \mathrm{C}$, curtain gas to $40 \mathrm{psi}$, the collision activated dissociation gas to "high", GS1 (nebulizer gas) to 45 psi and GS2 (heater gas) to $50 \mathrm{psi}$. The two MRM transitions which showed the best signal-to-noise ratios were monitored per analyte, the one with the highest signal intensity was selected as quantifier. Table 1 lists detailed HPLC-MS/MS parameters, and Fig. 1 shows a reconstructed HPLC-MS/MS chromatogram with the quantifier transition of each analyte. 
Table 1 Detailed scheduled multiple reaction monitoring (sMRM) parameters for all mycotoxins quantified

\begin{tabular}{|c|c|c|c|c|c|c|}
\hline Analyte & $\begin{array}{l}\text { Q1 mass } \\
{[\mathrm{m} / \mathrm{z}]^{\mathrm{a}}}\end{array}$ & $\begin{array}{l}\text { Q3 mass } \\
{\left[\mathrm{m} / \mathrm{z}^{\mathrm{a}}\right.}\end{array}$ & $\mathrm{DP}[\mathrm{V}]$ & $\mathrm{CE}[\mathrm{eV}]$ & $\begin{array}{l}\text { Exp. } t_{\mathrm{R}} \\
{[\mathrm{min}]^{\mathrm{b}}}\end{array}$ & $\begin{array}{l}\text { Mean } t_{\mathrm{R}} \pm \mathrm{SD} \\
{[\mathrm{min}]^{\mathrm{c}}}\end{array}$ \\
\hline $\mathrm{AFB}_{1}$ & $313[\mathrm{M}+\mathrm{H}]^{+}$ & $\begin{array}{l}241 \\
285\end{array}$ & 141 & $\begin{array}{l}51 \\
32\end{array}$ & 5.40 & $5.43 \pm 0.059$ \\
\hline $\mathrm{AFB}_{2}$ & $315[\mathrm{M}+\mathrm{H}]^{+}$ & $\begin{array}{l}287 \\
115\end{array}$ & 140 & $\begin{array}{l}37 \\
91\end{array}$ & 5.50 & $5.36 \pm 0.043$ \\
\hline $\mathrm{AFG}_{1}$ & $329[\mathrm{M}+\mathrm{H}]^{+}$ & $\begin{array}{l}243 \\
199\end{array}$ & $\begin{array}{l}35 \\
39\end{array}$ & $\begin{array}{l}37 \\
66\end{array}$ & 5.20 & $5.32 \pm 0.058$ \\
\hline $\mathrm{AFG}_{2}$ & $331[\mathrm{M}+\mathrm{H}]^{+}$ & $\begin{array}{l}245 \\
303\end{array}$ & 52 & $\begin{array}{l}41 \\
36\end{array}$ & 5.30 & $5.21 \pm 0.058$ \\
\hline $\mathrm{AFM}_{1}$ & $329[\mathrm{M}+\mathrm{H}]^{+}$ & $\begin{array}{l}273 \\
229\end{array}$ & 40 & $\begin{array}{l}34 \\
54\end{array}$ & 5.10 & $5.09 \pm 0.058$ \\
\hline ALT & $293[\mathrm{M}+\mathrm{H}]^{+}$ & $\begin{array}{l}197 \\
152\end{array}$ & 30 & $\begin{array}{l}39 \\
47\end{array}$ & 5.20 & $5.22 \pm 0.056$ \\
\hline AME & $271[\mathrm{M}-\mathrm{H}]^{-}$ & $\begin{array}{l}211 \\
213\end{array}$ & -44 & $\begin{array}{l}-45 \\
-50\end{array}$ & 6.35 & $6.30 \pm 0.106$ \\
\hline $\mathrm{AOH}$ & $257[\mathrm{M}-\mathrm{H}]^{-}$ & $\begin{array}{l}147 \\
171\end{array}$ & -80 & $\begin{array}{l}-38 \\
-40\end{array}$ & 5.50 & $5.50 \pm 0.056$ \\
\hline Bea & $784.4[\mathrm{M}+\mathrm{H}]^{+}$ & $\begin{array}{l}244.1 \\
262.2\end{array}$ & 5 & $\begin{array}{l}37 \\
36\end{array}$ & 8.60 & $8.59 \pm 0.053$ \\
\hline CIT & $251[\mathrm{M}+\mathrm{H}]^{+}$ & $\begin{array}{l}115 \\
91\end{array}$ & 75 & $\begin{array}{l}68 \\
54\end{array}$ & 6.40 & $6.50 \pm 0.107$ \\
\hline DH-CIT & $267[\mathrm{M}+\mathrm{H}]^{+}$ & $\begin{array}{l}231 \\
203\end{array}$ & 54 & $\begin{array}{l}32 \\
39\end{array}$ & 9.40 & $9.62 \pm 0.106$ \\
\hline DON & $297[\mathrm{M}+\mathrm{H}]^{+}$ & $\begin{array}{l}231 \\
219\end{array}$ & 70 & $\begin{array}{l}17 \\
17\end{array}$ & 3.50 & $3.18 \pm 0.117$ \\
\hline DON-3-GlcA & $471[\mathrm{M}-\mathrm{H}]^{-}$ & $\begin{array}{l}175 \\
265\end{array}$ & -200 & $\begin{array}{l}-38 \\
-38\end{array}$ & 4.50 & $4.55 \pm 0.112$ \\
\hline EnA & $704.4[\mathrm{M}+\mathrm{Na}]^{+}$ & $\begin{array}{l}350 \\
231.9\end{array}$ & 5 & $\begin{array}{l}68 \\
73\end{array}$ & 8.80 & $8.78 \pm 0.056$ \\
\hline $\mathrm{EnA}_{1}$ & $668.4[\mathrm{M}+\mathrm{H}]^{+}$ & $\begin{array}{l}210.1 \\
228.3\end{array}$ & 5 & $\begin{array}{l}35 \\
35\end{array}$ & 8.70 & $8.69 \pm 0.057$ \\
\hline EnB & $\begin{array}{l}640.4[\mathrm{M}+\mathrm{H}]^{+} \\
662.4[\mathrm{M}+\mathrm{Na}]^{+}\end{array}$ & $\begin{array}{l}196.2 \\
336.3\end{array}$ & $\begin{array}{l}50 \\
240\end{array}$ & $\begin{array}{l}32 \\
63\end{array}$ & 8.40 & $8.46 \pm 0.058$ \\
\hline $\mathrm{EnB}_{1}$ & $654.4[\mathrm{M}+\mathrm{H}]^{+}$ & $\begin{array}{l}214.2 \\
196.1\end{array}$ & 5 & $\begin{array}{l}35 \\
52\end{array}$ & 8.60 & $8.56 \pm 0.058$ \\
\hline $\mathrm{FB}_{1}$ & $722[\mathrm{M}+\mathrm{H}]^{+}$ & $\begin{array}{l}370 \\
334\end{array}$ & 125 & $\begin{array}{l}46 \\
54\end{array}$ & 4.90 & $4.91 \pm 0.035$ \\
\hline 10-OH-OTA & $420[\mathrm{M}+\mathrm{H}]^{+}$ & $\begin{array}{l}255 \\
181\end{array}$ & 65 & $\begin{array}{l}33 \\
61\end{array}$ & 5.70 & $5.78 \pm 0.064$ \\
\hline HT-2 & $\begin{array}{l}447\left[\mathrm{M}+\mathrm{Na}^{+}\right. \\
442\left[\mathrm{M}+\mathrm{NH}_{4}\right]^{+}\end{array}$ & $\begin{array}{l}345 \\
215\end{array}$ & $\begin{array}{l}140 \\
50\end{array}$ & $\begin{array}{l}25 \\
18\end{array}$ & 5.50 & $5.55 \pm 0.052$ \\
\hline HT-2-4-GlcA & $623[\mathrm{M}-\mathrm{H}]^{+}$ & $\begin{array}{l}345 \\
285\end{array}$ & 100 & $\begin{array}{l}39 \\
40\end{array}$ & 5.40 & $5.44 \pm 0.050$ \\
\hline $\mathrm{OTA} / 2^{\prime} R$-OTA & $404.1[\mathrm{M}+\mathrm{H}]^{+}$ & $\begin{array}{l}239 \\
102\end{array}$ & 70 & $\begin{array}{l}31 \\
88\end{array}$ & 6.70 & $\begin{array}{l}6.49 \pm 0.058^{\mathrm{d}} \\
6.69 \pm 0.064\end{array}$ \\
\hline OT $\alpha$ & $257[\mathrm{M}+\mathrm{H}]^{+}$ & $\begin{array}{l}102 \\
193\end{array}$ & 20 & $\begin{array}{l}54 \\
37\end{array}$ & 7.20 & $7.30 \pm 0.110$ \\
\hline $\mathrm{T}-2$ & $489[\mathrm{M}+\mathrm{Na}]^{+}$ & $\begin{array}{l}327 \\
387\end{array}$ & $\begin{array}{l}120 \\
145\end{array}$ & $\begin{array}{l}34 \\
29\end{array}$ & 6.10 & $6.15 \pm 0.082$ \\
\hline ZAN & $319[\mathrm{M}-\mathrm{H}]^{-}$ & $\begin{array}{l}205 \\
161\end{array}$ & -156 & $\begin{array}{l}-31 \\
-37\end{array}$ & 6.45 & $6.33 \pm 0.104$ \\
\hline ZEN & $317[\mathrm{M}-\mathrm{H}]^{-}$ & $\begin{array}{l}175 \\
131\end{array}$ & -140 & $\begin{array}{l}-32 \\
-37\end{array}$ & 6.45 & $6.42 \pm 0.102$ \\
\hline
\end{tabular}

MS transitions are listed for quantifier on top and qualifier below for each analyte. Expected retention time (Exp. $t_{\mathrm{R}}$ ) is equivalent to programmed time for sMRM algorithm and set against actual measured mean retention time (mean $t_{\mathrm{R}}$ ) followed by standard deviation (SD) of all analyzed calibration and recovery solutions

${ }^{\mathrm{a}}$ Quantifier top, qualifier below

${ }^{\mathrm{b}}$ Expected retention times, as they were programmed for scheduled MRM algorithm

${ }^{\mathrm{c}}$ Analyte mean retention time $\pm \mathrm{SD}[\mathrm{min}]$

${ }^{\mathrm{d}}$ OTA and 2'R-OTA show identical fragmentation patterns but are chromatographically separated with OTA eluting before $2{ }^{\prime} R$-OTA 


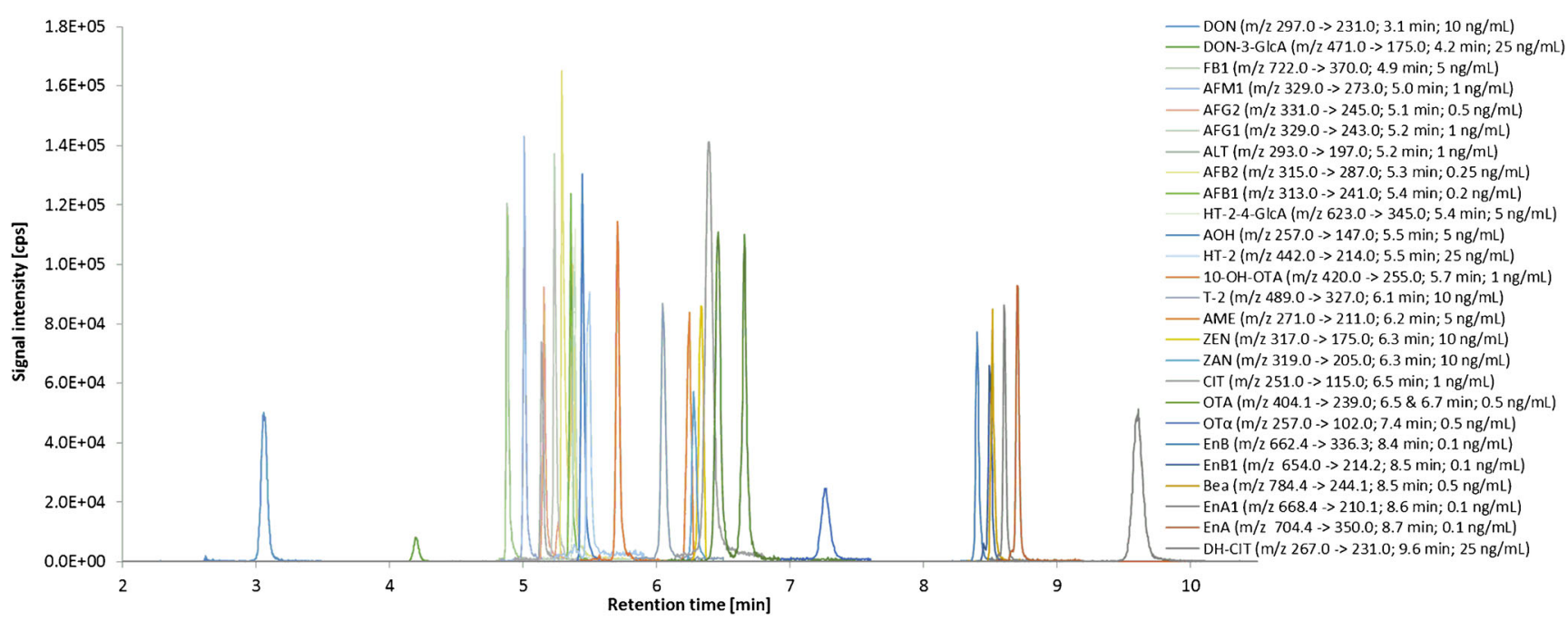

Fig. 1 Reconstructed HPLC-MS/MS chromatogram of whole blood (DBS) recovery samples spiked with 27 analytes. Quantifier transition and retention times are given in brackets for each signal

\section{Calibration, recovery, and stability testing}

Matrix-matched calibration was applied by adding standards to dried blood spot or dried serum spot extract. As no blood sample without detectable amounts of OTA and EnB was available, a sample with low natural contamination of both compounds was used to evaluate method parameters. OTA validation was performed using the diastereomer 2' $R$-OTA as previously described [21]. The level of EnB in the matrix solution was determined as $c_{\mathrm{EnB}}$, Matrix $=0.0361 \pm 0.0011 \mathrm{ng} / \mathrm{mL}$ via standard addition and taken into account for all further calculations.

Matrix-induced signal suppression and enhancement (SSE) was assessed by calculating the ratio of the slopes of matrixmatched and pure solvent calibration curve according to the following equation (see Tables 2 and 3):

$\mathrm{SSE}[\%]=\frac{\text { slope }_{\text {matrix-matched calibration }}}{\text { slope }_{\text {pure solvent calibration }}} * 100$

Values of $100 \%$ correspond to no impairment of analyte detection by matrix while SSE $>100 \%$ describes a signal enhancement due to a positive matrix effect and vice versa.

For every analyte, a calibration curve containing at least six points in the working range (Tables 2 and 3) was generated by dilution of the working solutions. Calibration solutions were freshly prepared for every measurement day. Reproducibility was determined by analyzing one real sample of the previously mentioned German sample cohort, naturally containing OTA, 2'R-OTA, and EnB, as quality control sample. The determination of LOD and LOQ was based on a signal to noise ratio $(\mathrm{S} / \mathrm{N})$ of 3 for LOD and $\mathrm{S} / \mathrm{N}$ of 10 for LOQ. The calculated LODs are given in Tables 2 and 3.

Recovery rates were determined by spotting blood and serum samples spiked with low, medium, and high analyte concentrations of the working range on DBS cards, followed by standard sample preparation procedure as described above. Three independent replicates for each concentration were prepared, analyzed, and quantified via the respective matrixmatched calibration for calculation of the recovery. Interday performance was evaluated by analysis of fortified recovery samples in both matrices on three different days. On the first day, the samples were worked up in triplicate and on the other 2 days in duplicate for all spiking levels. Detailed data of spiked concentrations, single and averaged results, and relative standard deviations are shown in Tables 2 and 3.

Stability testing of DBS/DSS was done with fortified samples which were stored at room temperature $\left(T=20^{\circ} \mathrm{C}\right)$ and in the dark, as these parameters are used for storage and shipping of DBS when pharmaceutical and medical analysis are applied. The samples were analyzed in duplicate for low, medium, and high spiking levels after storage for 1-10 weeks. Based on these values, mean relative degradation rates were calculated by comparison to freshly prepared recovery samples. Furthermore, two sets of spiked samples were prepared up to 24 weeks and stored at $4{ }^{\circ} \mathrm{C}$ in a fridge as well as in a freezer at $-18{ }^{\circ} \mathrm{C}$. The batches were kept in plastic boxes until analysis. Humidity during storage in laboratory and fridge was monitored by a hygro-/thermometer and was consistent at $60 \pm 10 \%$.

\section{Results and discussion}

In order to develop a multi-mycotoxin method based on dried blood spots (DBS) and dried serum spots (DSS), HPLC-MS/ MS analysis of 27 mycotoxins and metabolites was performed in positive and negative mode with electrospray ionization and scheduled multiple reaction monitoring ( $\left.\mathrm{SMRM}^{\mathrm{TM}}\right)$. 


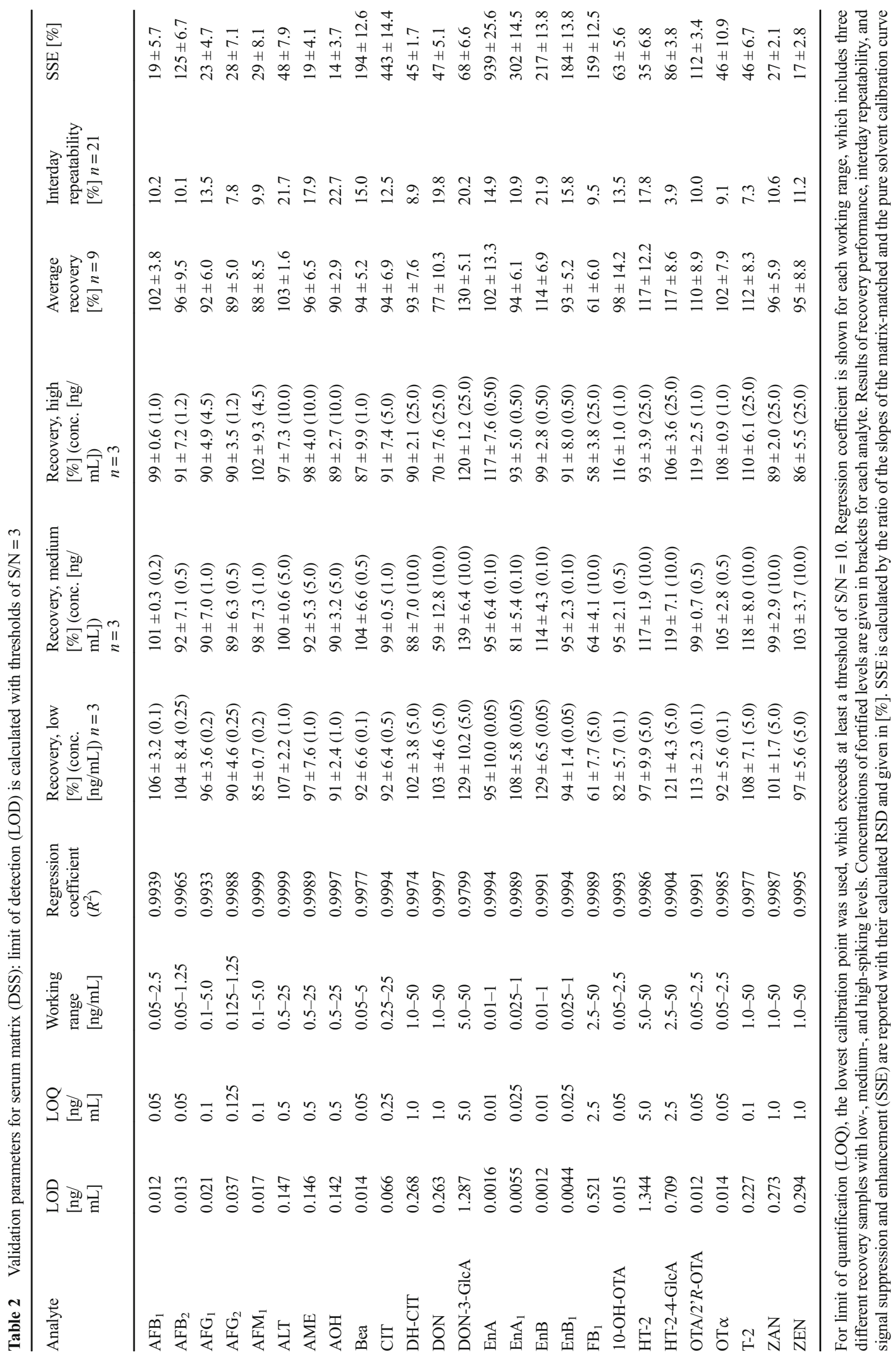




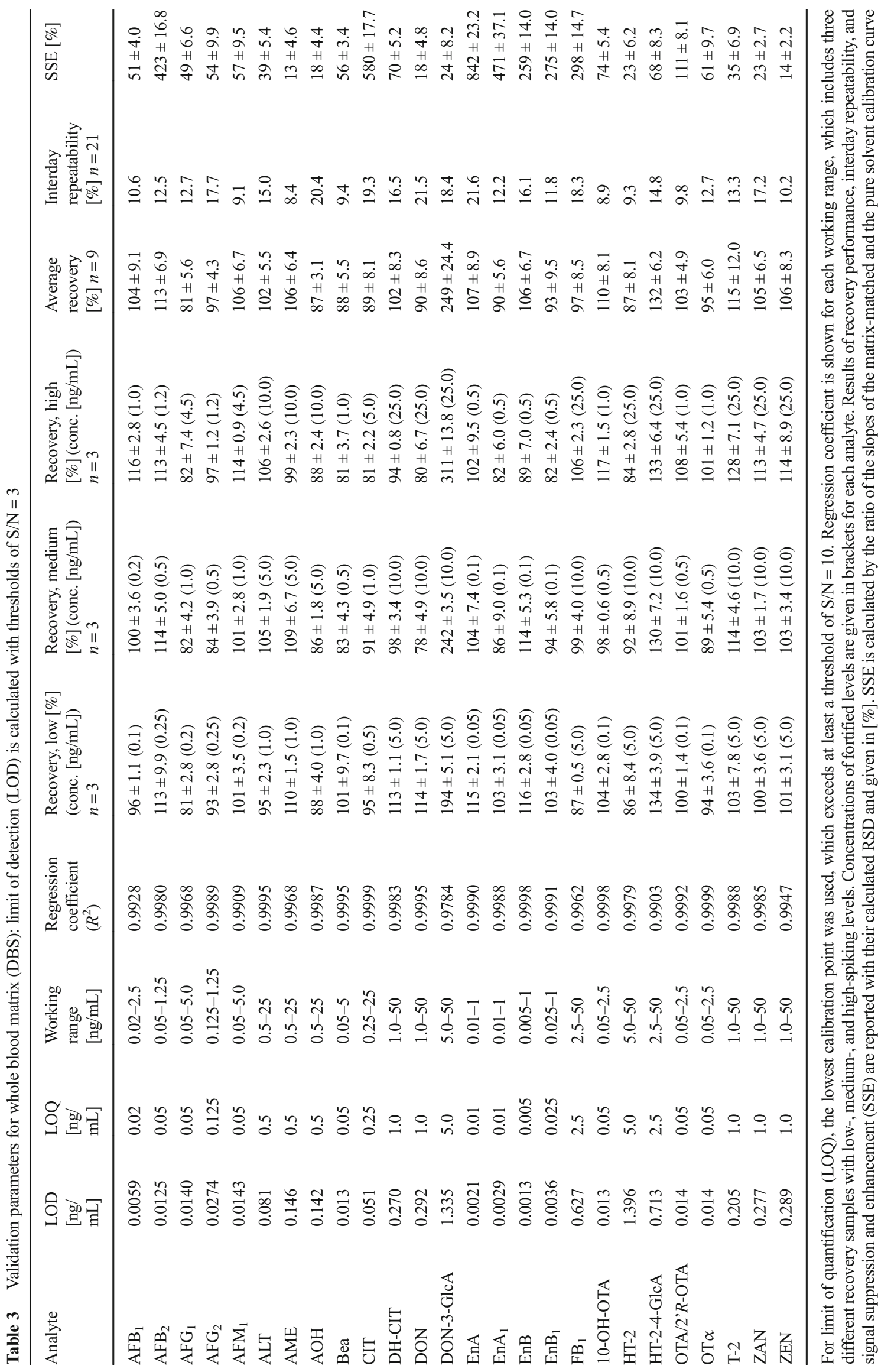


Analytes have been selected based on their occurrence, toxicological relevance, and availability [1, 23-29]. Polarity of the compounds was not considered as an important criterion for this study despite the fact that glucuronides are usually more relevant biomarkers for urinary exposure assessment than for blood-based human biomonitoring. However, kinetic studies in humans or animals might require the detection of these polar metabolites in order to investigate the metabolic activity and biotransformation rates of individuals.

\section{Sample preparation}

For sampling, the previously developed method [21] was extended and optimized to cover a larger set of 27 analytes and to include serum samples. A defined blood or serum volume of $100 \mu \mathrm{L}$ was spotted on standard filter paper cards, followed by drying, cutting the entire spot out of the paper, and an extraction with a mixture containing 35\% acetonitrile, 35\% acetone, and $30 \%$ water. After the extraction, an aliquot of the extract was evaporated to dryness, reconstituted, and centrifuged, leading to a clear and colorless solution. During optimization of the extraction procedure, it was observed that the applied centrifugal force has a strong impact on the subsequent HPLC-MS/MS analysis. Centrifugation at 22,000 $\times g$ was superior in matrix removal compared to the previously applied $3000 \times g$. For example, centrifugation at $22,000 \times g$ yielded a higher signal intensity and a lower $\mathrm{S} / \mathrm{N}$ ratio for $\mathrm{FB}_{1}$ (see Fig. S1, Electronic Supplementary Material (ESM)).

\section{Optimization of the HPLC-MS/MS conditions}

Special attention was paid to column selection, solvent additive and gradient to optimize peak shape, and signal response for all compounds from this heterogeneous group of analytes. Besides Nucleodur $\mathrm{C}_{18}$ Gravity SB, columns of the same sizes packed with ISIS, Pyramid and Gravity materials were evaluated. Matrix compounds in the extracted blood spot solutions strongly influence analyte separation while simultaneously interfering mass spectrometric detection by falsely triggering the measured transitions. Therefore, reducing the noise level closely to the expected retention times of the substances was mandatory. Another point was the baseline separation of OTA and $2^{\prime} R$-OTA within a short LC gradient as both isomers show the same MRM transitions. Both aims were preferable achieved by use of Gravity SB material when extracted matrix solutions spiked with the analytes were injected. The selected column material showed good retention properties for all analytes, in particular for OT $\alpha$ and DH-CIT which usually elute near the parent compounds OTA and CIT, respectively. Additionally, previously used formic acid as eluent additive was replaced by acetic acid. Despite being the weaker acid, chromatographic separation was still excellent. The $\mathrm{pH}$ gradient of $\mathrm{pH} 3.5$ to $\mathrm{pH}$ 2 caused by fortified concentrations of $0.1 \%$ acetic acid in water and $2 \%$ acetic acid in acetonitrile, respectively, led to improved peak shapes for DON as well as DH-CIT. Moreover, the weaker protonation properties resulted in higher ionization yields and therefore increased LODs. For instance, the chromatograms in Fig. S2 (ESM) demonstrate the influence of eluent additive on signal intensity and $\mathrm{S} / \mathrm{N}$ ratio.

Figure 1 shows a reconstructed HPLC-MS/MS chromatogram of spiked DBS recovery samples including all 27 compounds. All analytes and their phase-II-metabolites as well as OTA and 2'R-OTA were baseline separated to ensure no detection of artifacts. Chromatographic retention of the first eluting mycotoxin DON was adjusted to about $3.1 \mathrm{~min}$ in order to discard unwanted compounds by a diverter valve. Furthermore, to achieve maximum sensitivity with short dwell times, scheduled MRM was applied, reducing the detection window for every MRM to $30 \mathrm{~s}$ around the expected retention time.

Table 1 lists all detailed parameters for MS/MS detection. Moreover, programmed expected retention times are shown and set against those actually determined of all analyzed calibration and recovery standard solutions. During this analysis, over a period of 12 weeks, retention time shifts below $\pm 7 \mathrm{~s}$ were observed, providing the possibility to apply scheduled MRM to achieve maximum sensitivity with short dwell times. The windows for every MRM were set at $30 \mathrm{~s}$, except for the first and last eluting compounds DON and DH-CIT as it was expected that both would be the least accurately eluting. In fact, these analytes had some of the highest standard deviations concerning mean retention times as demonstrated in Table 1 . However, with a variation of about $\pm 6 \mathrm{~s}$ for DH-CIT, a retention time window of $30 \mathrm{~s}$ would have also been sufficiently broad.

Since matrix effects are a challenge in mass spectrometric detection, the aim was to obtain a matrix effect at a maximum of $10 \%$ of its intensity compared to neat eluent solutions in order to decrease the limit of detection as low as possible. Thus, an injection volume of $30 \mu \mathrm{L}$ compromises on absolute signal intensity as well as matrix suppression (Tables 2 and 3 ).

\section{Blood samples for matrix-matched calibration and quality control}

As no blank matrix solution was available, a blood sample with a low natural concentration of OTA and EnB was used for matrix-matched calibration. Figure 2 shows in sections A and $\mathrm{B}$ the extracted ion traces for both analytes in whole blood and serum matrix. The used matrix solution lacked the presence of $2^{\prime} R$-OTA, which was used for the determination of validation parameters of OTA. Chromatograms C and D in Fig. 2 show the ion traces of a human blood sample containing OTA, $2^{\prime} R$ $\mathrm{OTA}$, and $\mathrm{EnB}$ which was used as quality control sample.

The diastereomers OTA and 2'R-OTA are baseline separated and show the same ratio of the signal transition intensities in both processed whole blood and serum samples matrices. Reproducibility was investigated by the analysis of control 
Fig. 2 Extracted ion chromatograms of OTA/2' $R$-OTA (yellow transition $\mathrm{m} / \mathrm{z}$ 404.1 $\rightarrow$ 239.0, blue transition $\mathrm{m} / \mathrm{z}$ $404.1 \rightarrow 102.0)$ and EnB (green transition $\mathrm{m} / \mathrm{z}, 640.4 \rightarrow 196.2$, orange transition $\mathrm{m} / \mathrm{z}, 662.4 \rightarrow$ 336.3) in whole blood (a and $\mathbf{c})$ and serum (b and d). a, b Analyte signals for the used matrix solutions containing $0.291 \mathrm{ng} / \mathrm{mL}$ OTA and $0.0349 \mathrm{ng} / \mathrm{mL}$ EnB. c, $\mathbf{d}$ A real sample which was used as quality control sample containing $0.283 \mathrm{ng} / \mathrm{mL}$ OTA, $0.193 \mathrm{ng} / \mathrm{mL}$ 2' $R$ OTA, and $0.0366 \mathrm{ng} / \mathrm{mL} \mathrm{EnB}$
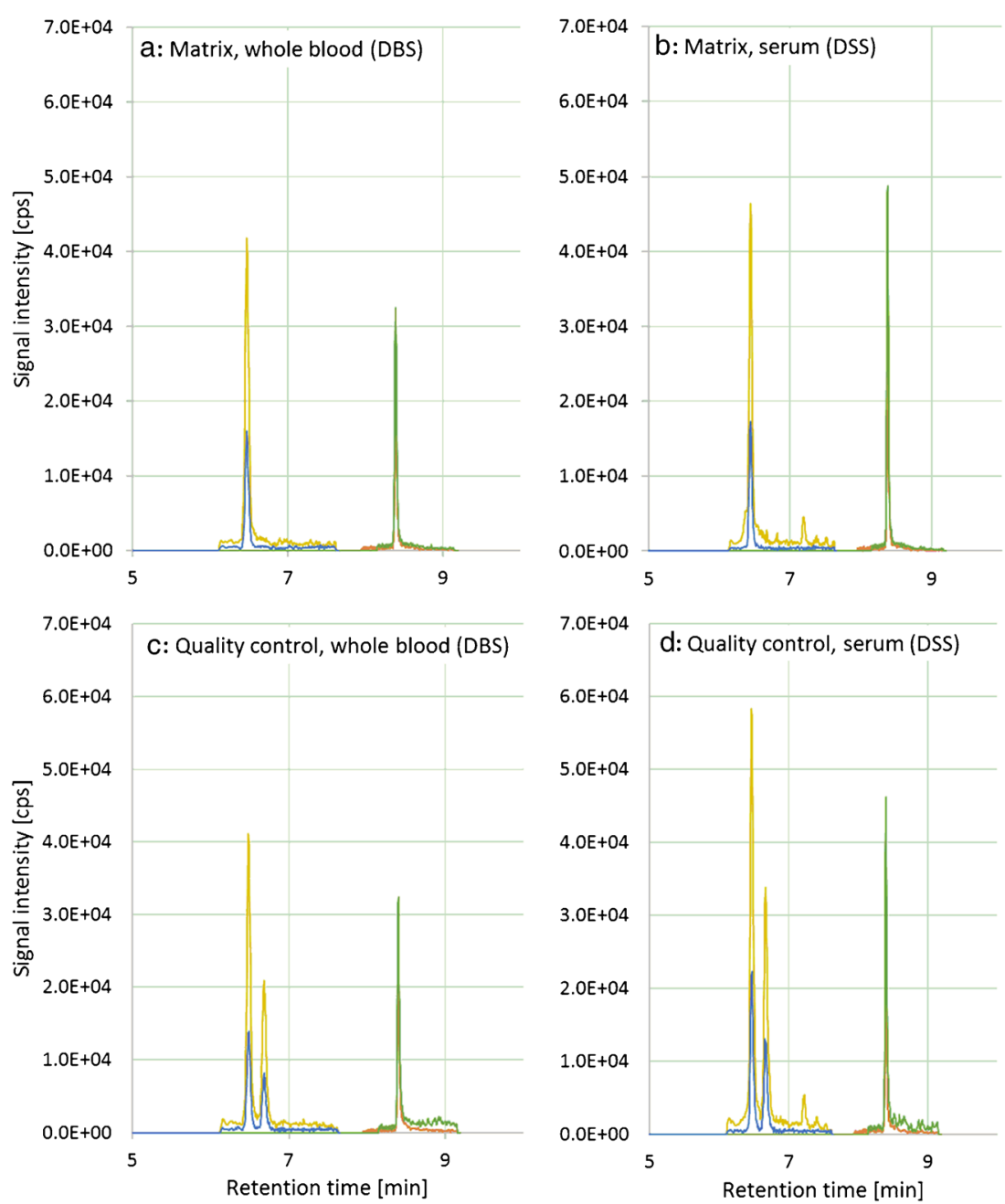

samples over a period of 7 days for whole blood and serum. For this, a sample naturally contaminated with OTA, 2' $R$ OTA, and EnB was chosen for performance control in order to provide results most closely to available samples. Therefore, quality control samples were freshly prepared and analyzed on each measurement day and reproducibility was accepted with $7.5 \%$ for OTA, $7.2 \%$ for 2'R-OTA, and $4.2 \%$ for EnB, consequently.

\section{Validation results}

Validation experiments were carried out by analyzing linearity, matrix effects, recovery, limit of detection (LOD), and limit of quantification (LOQ) for the serum (Table 2) and whole blood (Table 3) as matrices. Determination of LOD and LOQ was made via linear regression of mean signal to noise ratios of the four lowest matrix calibration standards of each compound. Matrix effect (SSE) calculation was done by comparison of signal intensities in aqueous and matrix solutions by means of the slopes of both calibration curves (see Experimental section for details). Furthermore, the chromatograms indicate no distinct difference between the two matrices concerning the noise levels around the signals (Fig. 2).

Best limits of detection were achieved for the enniatins at about $0.001-0.006 \mathrm{ng} / \mathrm{mL}$ in both matrices. Next, the aflatoxins, beauvericin, CIT, 10-OH-OTA, OT $\alpha$, and OTA/2'ROTA showed good LODs with calculated values up to $0.015 \mathrm{ng} / \mathrm{mL}$. Poorest responses have been determined for HT-2 and DON-3-GlcA with about 1.3-1.4 ng/mL for both analytes. Regression coefficients $\left(R^{2}\right)$ of 0.9999 to 0.9784 were accomplished by linear regression analysis. Maximum calibration concentrations were set at $50 \mathrm{ng} / \mathrm{mL}$ as higher blood contaminations were not anticipated. The LOQ values obtained with this new method indicate that the DBS-based sample preparation technique in combination with modern mass spectrometers is able to reach LOQ values comparable to those of previously published analyte-specific methods. For example, current OTA detection methods based on liquid-liquid extraction of the plasma, urine, or human breast milk lead to similar LOQs of $0.03 \mathrm{ng} / \mathrm{mL}$ [39]. Moreover, quantification of CIT can be carried out by immunoaffinity column cleanup resulting in a LOQ of $0.15 \mathrm{ng} / \mathrm{mL}$ for blood plasma samples [7]. Analysis of 
beauvericin and enniatins in pig plasma using HPLC-MS/MS after solid phase extraction reached LOQs of $0.1-0.2 \mathrm{ng} / \mathrm{mL}$ which are about one decade higher than the values achieved here (Table 3). Similar method parameters were acquired when human plasma samples were analyzed [5].

Recovery rates were determined by spiking blood and serum samples with low, medium, and high analyte concentrations (see Tables 2 and 3 for spiking levels). As shown in Tables 2 and 3, average recovery rates of 80 to $120 \%$ were achieved for 24 out of 27 mycotoxins in the serum and whole blood. $\mathrm{FB}_{1}$ and $\mathrm{DON}$ reached lower recovery rates of $61 \pm 6.0$ and $77 \pm 10.3 \%$, whereas DON-3-GlcA showed a slightly higher recovery rate of $130 \pm 5.1 \%$ in serum matrix. In whole blood, the lowest recovery rate was obtained for $\mathrm{AFG}_{1}$ with $81 \pm 5.6 \%$ and the highest with $132 \pm 6.2 \%$ for HT-2-4-GlcA.

For DON-3-GlcA, the determined mean recovery in DBS was $249 \%$, which is out of the range for reliable quantitative analysis. The high polarity of this compound as well as the low slope of the linear calibration curve due to strong matrix effects are possible explanations. Carry-over effects through the injection system could be excluded as injections of blank solutions after the highest standards were always negative for all compounds. Besides DON-3-GLcA, also DON-15-GlcA is reported as main metabolite of DON in humans [28, 40-43]. Both compounds coelute on most chromatographic systems and show mostly identical fragmentation behavior. As only DON-3-GlcA was available, this was used as reference and an accidental detection of coeluting DON-15-GlcA was accepted.

Matrix effects due to signal suppression or enhancement are a major concern and a well-known phenomenon in mass spectrometry. Consequently, its consideration is recommended for the establishment of new analytical methods. For example, matrix suppression down to $40 \%$ signal intensity for CIT can be determined in urine samples even after liquidliquid extraction followed by solid phase extraction [44]. Therefore, high signal suppression due to only little matrix removal was expected and observed for most of the analytes in both matrices, as it is often described for multi-analyte biomonitoring methods $[11,45]$.

Among all compounds $\mathrm{AOH}$ undergoes the strongest matrix effect in whole blood resulting in a signal intensity of only $14 \%$ compared to the standard solution in neat solvent. The lowest signal intensity in the serum was observed for alternariol monomethyl ether which was only $13 \%$ compared to the standard solution. OTA and 2'R-OTA show in both matrices only minor alteration concerning signal intensity compared to blank solutions. In contrast, $\mathrm{FB}_{1}$, CIT, and the enniatins undergo strong signal enhancement, which has particularly for EnB already been reported [46]. Enniatins stand out with positive matrix effects of up to $939 \%$ for EnA in processed serum matrix. Interestingly, beauvericin shows a decent signal suppression when detected in whole blood and an increased signal intensity in serum matrix (SSE of 56 and $194 \%$, respectively).

Although the levels of SSEs are severe for some analytes, the matrix effects are highly reproducible as can be seen by their relative standard deviations (RSD). For example, calibration curves for EnA and AME are shown in Fig. S3 (ESM). For the calculations of RSD, five sets of matrix-matched calibration and neat solvent calibration curves were used (Tables 2 and 3). As a result, only slight differences between both matrices were observed for most analytes. Endogenous blood compounds are assumed to be co-extracted and to interfere mass spectrometric detection. Due to the lack of blood cells, we expected a lower matrix influence of serum compared to whole blood; however, the results do not support this. Erythrocytes appear to be intact but are distorted from dehydration in DBS samples [47]. However, it can be supposed that cells are destroyed during the extraction procedure by applying organic solvents as well as sonication. In fact, not all analytes show comparable SSE in both extracted matrices besides beauvericin. Nevertheless, determined recovery rates are comparable for both matrices. In order to study any influence of different matrix samples on DBS analysis, five randomly chosen blood matrices from the study cohort $(n=50)$ were fortified with the highest spiking level in triplicate. The results are shown in ESM Table S1 and no significant differences regarding the average recovery rate compared to the prior obtained results were observed $(P>0.05)$. In summary, the developed method achieves reliable validation parameters for mycotoxin detection in DSS and DBS including a broad range of analyte polarities. The developed method accomplishes a stable and robust quantification performance by use of matrix-matched calibration without the need of isotopically labelled standards, which are not commercially available for most of the mycotoxin metabolites as internal standards.

\section{Stability testing}

A crucial point in DBS and DSS analysis is the stability of the analytes on the filter cards. Especially as DBS cards are usually handled and shipped at room temperature, a certain ageing of the sample material can be expected. Thus, the next step in method validation was stability testing of the analytes in DBS and DSS matrices. On the basis of fortified recovery samples, relative recovery rates were investigated by storing the DSS and DBS cards in the dark at room temperature $(T=$ $20^{\circ} \mathrm{C}$ ), at $4{ }^{\circ} \mathrm{C}$ as well as $-18^{\circ} \mathrm{C}$.

The stability of all 27 compounds was analyzed and the results after storage for 1,5 , and 10 weeks at room temperature and after storage for 24 weeks at 4 and $-18{ }^{\circ} \mathrm{C}$ are summarized in Table 4 for whole blood (the results for serum can be found in Table S2 (ESM) as no clear difference between both DBS and DSS matrix was observed when samples were stored at 4 or $-18{ }^{\circ} \mathrm{C}$ ). Detailed relative recovery rates for storage at 
Table 4 Average relative analyte concentration $\pm \operatorname{RSD}[\%]$ of mycotoxins when stored as dried blood spots for 1, 5, and 10 weeks at room temperature and 24 weeks at 4 and $-18{ }^{\circ} \mathrm{C}$ in the dark

Whole blood (DBS)

\begin{tabular}{|c|c|c|c|c|c|}
\hline Temperature & & $20^{\circ} \mathrm{C}$ & & $4{ }^{\circ} \mathrm{C}$ & $-18{ }^{\circ} \mathrm{C}$ \\
\hline Storage time & 1 week & 5 weeks & 10 weeks & 24 weeks & 24 weeks \\
\hline AFB1 & $83.8 \pm 7.7$ & $66.9 \pm 7.5$ & $44.7 \pm 1.7$ & $79.2 \pm 10.4$ & $95.1 \pm 8.0$ \\
\hline AFB2 & $98.3 \pm 3.4$ & $44.4 \pm 1.7$ & $16.7 \pm 1.5$ & $85.1 \pm 17.8$ & $96.8 \pm 5.6$ \\
\hline AFG1 & $98.2 \pm 3.9$ & $76.0 \pm 7.9$ & $59.9 \pm 4.2$ & $86.8 \pm 2.1$ & $102.0 \pm 7.6$ \\
\hline AFG2 & $93.0 \pm 4.1$ & $69.7 \pm 7.4$ & $44.7 \pm 3.2$ & $88.2 \pm 5.0$ & $94.6 \pm 5.2$ \\
\hline AFM1 & $82.6 \pm 7.6$ & $58.2 \pm 7.4$ & $35.7 \pm 1.7$ & $91.1 \pm 5.7$ & $95.1 \pm 3.8$ \\
\hline ALT & $65.0 \pm 12.0$ & $36.5 \pm 5.9$ & $21.2 \pm 6.4$ & $76.1 \pm 8.1$ & $91.2 \pm 7.7$ \\
\hline AME & 89.66 .1 & $54.2 \pm 6.4$ & $10.3 \pm 6.7$ & $85.1 \pm 7.1$ & $96.3 \pm 5.2$ \\
\hline $\mathrm{AOH}$ & $63.5 \pm 10.7$ & $30.1 \pm 9.4$ & $5.6 \pm 7.2$ & $86.8 \pm 10.8$ & $103.7 \pm 7.5$ \\
\hline Bea & $91.0 \pm 3.0$ & $67.4 \pm 9.2$ & $47.0 \pm 5.1$ & $97.9 \pm 12.7$ & $101.1 \pm 10.0$ \\
\hline CIT & $75.4 \pm 3.6$ & $25.3 \pm 7.1$ & $19.4 \pm 2.3$ & $79.9 \pm 4.0$ & $96.5 \pm 9.6$ \\
\hline DH-CIT & $44.7 \pm 7.5$ & $9.9 \pm 2.4$ & $4.7 \pm 1.9$ & $90.9 \pm 10.6$ & $100.5 \pm 12.5$ \\
\hline DON & $94.5 \pm 8.6$ & $64.1 \pm 9.8$ & $31.9 \pm 6.3$ & $85.6 \pm 6.7$ & $94.0 \pm 9.6$ \\
\hline DON-3-GICA & $77.7 \pm 8.0$ & $47.7 \pm 3.0$ & $39.4 \pm 8.0$ & $84.3 \pm 13.8$ & $92.8 \pm 10.4$ \\
\hline EnA & $99.2 \pm 2.4$ & $83.7 \pm 6.5$ & $46.2 \pm 2.7$ & $78.5 \pm 7.3$ & $97.8 \pm 9.4$ \\
\hline EnA1 & $96.0 \pm 1.5$ & $82.7 \pm 1.4$ & $60.3 \pm 1.1$ & $81.1 \pm 6.8$ & $95.9 \pm 8.7$ \\
\hline EnB & $98.0 \pm 2.8$ & $91.9 \pm 6.9$ & $75.0 \pm 4.7$ & $79.7 \pm 7.9$ & $91.9 \pm 8.2$ \\
\hline EnB1 & $90.8 \pm 3.6$ & $81.2 \pm 3.0$ & $57.8 \pm 1.3$ & $84.2 \pm 5.6$ & $101.8 \pm 9.4$ \\
\hline FB1 & $80.7 \pm 7.2$ & $55.3 \pm 5.6$ & $36.7 \pm 5.9$ & $82.5 \pm 5.9$ & $94.8 \pm 3.1$ \\
\hline 10-ОН-ОТА & $90.6 \pm 3.1$ & $72.8 \pm 11.9$ & $68.9 \pm 4.4$ & $81.6 \pm 4.2$ & $102.6 \pm 6.3$ \\
\hline HT-2 & $96.1 \pm 10.1$ & $69.4 \pm 5.1$ & $53.8 \pm 5.6$ & $83.7 \pm 6.6$ & $90.2 \pm 5.6$ \\
\hline HT-2-4-GIcA & $86.0 \pm 5.6$ & $71.0 \pm 9.0$ & $50.3 \pm 7.3$ & $85.2 \pm 9.6$ & $91.8 \pm 9.0$ \\
\hline OTA $/ 2^{\prime} R$-ОTA & $99.3 \pm 4.0$ & $93.2 \pm 4.9$ & $89.3 \pm 5.6$ & $90.2 \pm 5.0$ & $95.3 \pm 4.8$ \\
\hline OT $\alpha$ & $84.0 \pm 3.7$ & $56.5 \pm 12.2$ & $35.5 \pm 5.9$ & $83.5 \pm 11.5$ & $95.9 \pm 8.3$ \\
\hline $\mathrm{T}-2$ & $76.5 \pm 6.7$ & $38.9 \pm 5.0$ & $15.1 \pm 2.2$ & $88.3 \pm 10.3$ & $91.0 \pm 8.9$ \\
\hline ZAN & $83.6 \pm 10.7$ & $55.2 \pm 9.0$ & $31.9 \pm 6.4$ & $91.3 \pm 17.2$ & $96.8 \pm 14.1$ \\
\hline ZEN & $89.0 \pm 8.7$ & $29.9 \pm 9.6$ & $8.3 \pm 4.9$ & $82.2 \pm 6.2$ & $90.3 \pm 8.8$ \\
\hline
\end{tabular}

Freshly prepared recovery solutions were set at $100 \%$ and corresponding recovery rate after storage are depicted. Color gradient emphasizes the level of nearly no degradation $(\geq 95 \%$, green) towards the highest $(\leq 50 \%$, red) with color steps of $5 \%$

$20^{\circ} \mathrm{C}$ for $1-10$ weeks are shown in Figs. S4 and S5 (ESM). Besides OTA/2'R-OTA, all other compounds showed a timedependent degradation at room temperature. Nearly all mean recovery rates decrease already within the first week after sample collection (Table 4). The enniatins, HT-2/HT-2-4GlcA as well as 10-OH-OTA and OT $\alpha$ show a moderate reduction over the 10-week period resulting in remaining toxin concentrations of $37-75 \%$ after 10 weeks. The other compounds undergo a relatively fast degradation under these typical storage conditions to only 4-37\% of the original concentration. This trend can be observed for both matrices, DBS as well as DSS (for the DSS results see Table S2, ESM). However, storage for shorter periods like 1 week at room temperature, a time frame sufficient for typical sample shipping, still provides recoveries of $61 \%$ for DH-CIT, $63 \%$ for $\mathrm{AFG}_{1}$, and for all other compounds at least $74 \%$ in serum. In whole blood, after 1 week only DH-CIT (45\%), AOH (64\%), and ALT $(65 \%)$ show higher degradation, while all other mycotoxins or metabolites show at least a recovery of $75 \%$ of the original concentration.
Although room temperature is the typical condition for shipping and storage of DBS in clinical studies, the relative recovery rates of fortified DBS and DSS were investigated when the samples were stored at 4 and $-18{ }^{\circ} \mathrm{C}$ up to 24 weeks. As representative example, stability curves for $\mathrm{AFB}_{1}$ under all storage conditions are shown in Fig. 3. The summarized results in Table 4 clearly demonstrate that most analytes are relatively stable when stored at 4 or $-18{ }^{\circ} \mathrm{C}$. After 24 weeks of storage, all recovery rates were still above 76 and $88 \%$ at 4 and $-18{ }^{\circ} \mathrm{C}$, respectively. During all studies, humidity was held at a constant level of $60 \pm 10 \%$, which corresponds to typical laboratory conditions.

Thus, we recommend to store the dried samples under light exclusion in sealed containers at $-18^{\circ} \mathrm{C}$ to minimize potential degradation of the mycotoxins as discussed above. To our knowledge, this is the first evaluation of mycotoxin stability in dried physiological samples such as DBS and DSS. The results clearly show that sample storage under optimal conditions is safe but can become an issue when temperature above $4{ }^{\circ} \mathrm{C}$ is reached. 


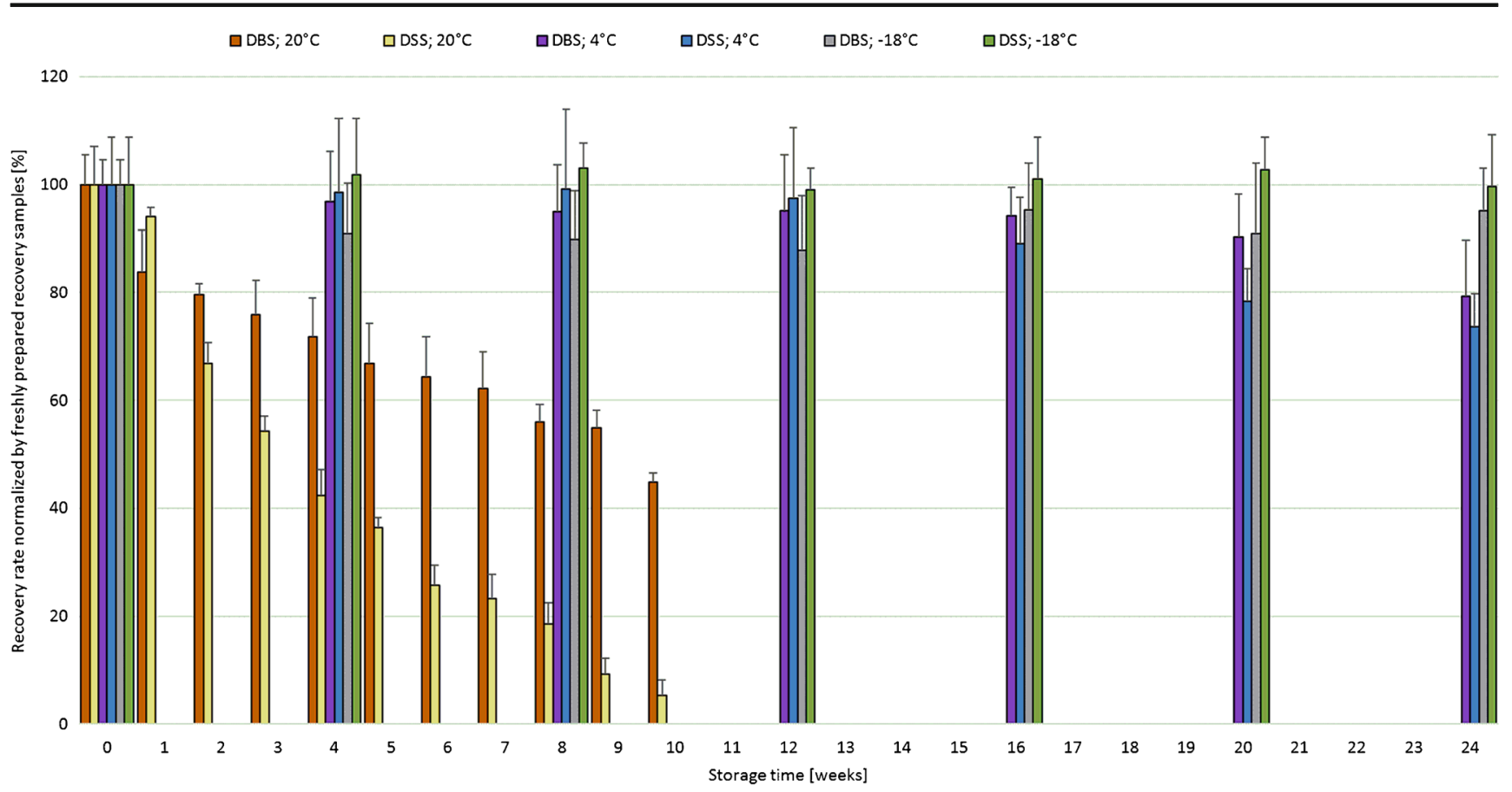

Fig. 3 Relative recovery rates of $\mathrm{AFB}_{1}$ in fortified DBS and DSS; storage at room temperature $\left(20^{\circ} \mathrm{C}\right)$ for $1-10$ weeks and at $4{ }^{\circ} \mathrm{C}$ as well as $-18{ }^{\circ} \mathrm{C}$ for 4-24 weeks

\section{Biomonitoring of blood samples}

The developed multi-mycotoxin method was applied for the analysis of blood samples of a German sample cohort which have already been analyzed in our first DBS-study for OTA and 2'R-OTA [21]. For multi-mycotoxin analysis of this sample cohort, DBS $(n=50)$ were prepared in duplicate and quantified according to the matrix-matched calibration parameters. Besides OTA and EnB, no other mycotoxins or metabolites were detectable. Although DON and especially its phase-II-metabolite are often detectable in human urine samples from Germany [11], it seems that DON is rapidly converted and excreted as it is not detectable in DBS samples. Figure 2 shows typical HPLC-MS/ MS chromatograms as the quality control is one sample out of the German sample cohort.

Enniatin B was detectable in all samples with a mean value of $0.0367 \pm 0.0179 \mathrm{ng} / \mathrm{mL}$ and a median of $0.0330 \mathrm{ng} / \mathrm{mL}$. The lowest and highest concentrations were with 0.0144 $0.1071 \mathrm{ng} / \mathrm{mL}$ in the calibration range. Intake of EnB by food is supposed to be the main reason of exposure. However, risk assessment is currently not possible due to the lack of data for dietary exposure as well as toxicity, so that no TDI is established yet [48]. Considering the low concentrations of EnB in the analyzed DBS, this study contributes results for the presence of EnB in human blood samples as it has already been reported in urine and breast milk samples [11, 25]. Previously quantified OTA and 2'R-OTA [21] were reanalyzed by this multi-mycotoxin method. OTA was found in all samples as well as $2^{\prime} R$-OTA in the same samples as before. However, some samples $(n=9$ out of 34$)$ showed signals for 2' $R$-OTA between LOD and LOQ due to slightly lower sensitivity of this multi-mycotoxin method. Therefore, all positive findings were confirmed. Figure S6 (ESM) demonstrates the correlation between the analyte amounts calculated by both methods. The arithmetic mean of OTA $(n=50)$ by [21] with $c_{\mathrm{OTA}}=0.211 \pm 0.064 \mathrm{ng} / \mathrm{mL}$ is matching to $c_{\mathrm{OTA}}=0.207 \pm 0.063 \mathrm{ng} / \mathrm{mL}$. Likewise, the average values for $2^{\prime} R$-OTA are $c_{2}{ }^{\prime} R$-OTA $=0.112 \pm 0.092 \mathrm{ng} / \mathrm{mL}(n=34)$ and $c_{2}{ }^{\prime}$-OTA $=0.143 \pm 0.100 \mathrm{ng} / \mathrm{mL} \quad(n=25)$, respectively. Latter value is certainly higher because the amounts of nine samples which show signals $>$ LOD are not considered. Calculated regression coefficients of $R^{2}=0.9043$ (OTA) and $R^{2}=0.9512\left(2^{\prime} R\right.$-OTA) support the comparability of both sample preparations (Fig. S6, ESM). Thus, the results clearly demonstrate the comparability of both methods for the detection and quantification of OTA and 2'R-OTA in DBS.

\section{Conclusion}

A DBS- and DSS-based sample preparation technique for the detection of various mycotoxins and metabolites was established and validated. The developed multi-mycotoxin method allows the simultaneous detection and quantification of 27 analytes by HPLC-MS/MS in dried whole blood (DBS) and serum spots (DSS). On the basis of previous DBS studies $[21,22]$, the improved method has the same advantages such as simplified storage and shipment conditions as well as reduced use of chemicals and materials compared to other sampling techniques. 
The developed DSS and DBS-based method was evaluated for linearity, limit of detection, and quantification, robustness, recovery, and stability of fortified samples. It could be shown that DSS and DBS are a suitable sampling technique for the detection of multi-mycotoxin exposure due to adequate validation parameters for most of the incorporated mycotoxins and metabolites. Complications arise from stability issues which have to be taken into account and for this reason storage of samples at $-18^{\circ} \mathrm{C}$ is recommended. The developed method was applied to DBS samples of a German cohort, showing that besides OTA [21], all samples $(n=50)$ were positive for EnB with mean levels of $0.0367 \mathrm{ng} / \mathrm{mL}$. All in all, a novel application for the use of DSS and DBS for multi-mycotoxin exposure studies was developed and validated, proofing the effective performance characteristics needed for biomarker or biomonitoring approaches. As some mycotoxins or metabolites are mainly detectable in urine (e.g., DON-3-GlcA) [10, $11]$ and others such as OTA, 2' $R$-OTA, EnB mainly in the blood (Fig. 2), the analysis of both matrices is recommended to evaluate the human and animal exposure to mycotoxins. Besides dilute-and-shoot approaches used for urine samples, the use of DBS and DSS provides an optimal extension of human and animal biomonitoring due to the non-invasive sample collection and easy sample preparation.

\section{Compliance with ethical standards}

Conflict of interest The authors declare that they have no conflict of interests.

Open Access This article is distributed under the terms of the Creative Commons Attribution 4.0 International License (http:// creativecommons.org/licenses/by/4.0/), which permits unrestricted use, distribution, and reproduction in any medium, provided you give appropriate credit to the original author(s) and the source, provide a link to the Creative Commons license, and indicate if changes were made.

\section{References}

1. Bennett JW, Klich M. Mycotoxins. Clin Microbiol Rev. 2003;16: 497-516.

2. van Egmond HP, Schothorst RC, Jonker MA. Regulations relating to mycotoxins in food. Anal Bioanal Chem. 2007;389:147-57.

3. Marin S, Ramos AJ, Cano-Sancho G, Sanchis V. Mycotoxins: occurrence, toxicology, and exposure assessment. Food Chem Toxicol. 2013;60:218-37.

4. Turner PC, Flannery B, Isitt C, Ali M, Pestka J. The role of biomarkers in evaluating human health concerns from fungal contaminants in food. Nutr Res Rev. 2012;25:162-79.

5. Serrano AB, Capriotti AL, Cavaliere C, Piovesana S, Samperi R, Ventura S, et al. Development of a rapid LC-MS/MS method for the determination of emerging Fusarium mycotoxins. Toxins. 2015;7: 3554-71.

6. Muñoz K, Blaszkewicz M, Degen GH. Simultaneous analysis of ochratoxin A and its major metabolite ochratoxin alpha in plasma and urine for an advanced biomonitoring of the mycotoxin. $\mathrm{J}$ Chromatogr B. 2010;878:2623-9.
7. Blaszkewicz M, Muñoz K, Degen GH. Methods for analysis of citrinin in human blood and urine. Arch Toxicol. 2013;87:1087-94.

8. Torres O, Matute J, Gelineau-van Waes J, Maddox JR, Gregory SG, Ashley-Koch AE, et al. Urinary fumonisin B1 and estimated fumonisin intake in women from high- and low-exposure communities in Guatemala. Mol Nutr Food Res. 2014;58:973-83.

9. Vettorazzi A, González-Peñas E, de Cerain AL. Ochratoxin A kinetics: a review of analytical methods and studies in rat model. Food Chem Toxicol. 2014;72:273-88.

10. Warth B, Sulyok M, Krska R. LC-MS/MS-based multibiomarker approaches for the assessment of human exposure to mycotoxins. Anal Bioanal Chem. 2013;405:5687-95.

11. Gerding J, Cramer B, Humpf H-U. Determination of mycotoxin exposure in Germany using an LC-MS/MS multibiomarker approach. Mol Nutr Food Res. 2014;58:2358-68.

12. Stove CP, Ingels ASME, De Kesel PMM, Lambert WE. Dried blood spots in toxicology: from the cradle to the grave? Crit Rev Toxicol. 2012;42:230-43.

13. Mercolini L, Mandrioli R, Sorella V, Somaini L, Giocondi D, Serpelloni G, et al. Dried blood spots: liquid chromatographymass spectrometry analysis of $\Delta 9$-tetrahydrocannabinol and its main metabolites. J Chromatogr A. 2013;1271:33-40.

14. Batterman S, Chernyak S. Performance and storage integrity of dried blood spots for PCB, BFR and pesticide measurements. Sci Total Environ. 2014;494-495:252-60.

15. Shlosberg A, Rumbeiha WK, Lublina A, Kannan K. A database of avian blood spot examinations for exposure of wild birds to environmental toxicants: the DABSE biomonitoring project. J Environ Monit. 2011;13:1547-58.

16. Knudsen R, Slazyk W, Richmond J, Hannon W. CDC Guidelines for the shipment of dried blood spot specimens. 2012. https:/www. cdc.gov/labstandards/pdf/nsqap/Bloodspot_Transportation Guidelines.pdf Accessed 12 July 2016.

17. Blumenfeld TA, Hertelendy WG, Ford SH. Simultaneously obtained skin-puncture serum, skin-puncture plasma, and venous serum compared, and effects of warming the skin before puncture. Clin Chem. 1977;23:1705-10.

18. Kiyoshi M, Keiko N, Minoru K, Toshiaki O, Tsuneo T. An improved assay of thyrotropin in dried blood samples on filter paper as a screening test for neonatal hypothyroidism. Clin Chim Acta. 1976;73:241-9.

19. O'Broin S, Gunter E. Dried-serum spot assay for folate. Clin Chem. 2002;48:1128-30.

20. Desbois D, Roque-Afonso AM, Lebraud P, Dussaix E. Use of dried serum spots for serological and molecular detection of hepatitis a virus. J Clin Microbiol. 2009;47:1536-42.

21. Cramer B, Osteresch B, Muñoz KA, Hillmann H, Sibrowski W, Humpf $\mathrm{H}-\mathrm{U}$. Biomonitoring using dried blood spots: detection of ochratoxin A and its degradation product 2'R-ochratoxin A in blood from coffee drinkers. Mol Nutr Food Res. 2015;59:1837-43.

22. Osteresch B, Cramer B, Humpf HU. Analysis of ochratoxin A in dried blood spots - correlation between venous and finger prick blood, the influence of hematocrit and spotted volume. J Chromatogr B. 2016;1020:158-64.

23. Ostry V. Alternaria mycotoxins: an overview of chemical characterization, producers, toxicity, analysis and occurrence in foodstuffs. World Mycotoxin J. 2008;1:175-88.

24. Sy-Cordero AA, Pearce CJ, Oberlies NH. Revisiting the enniatins: a review of their isolation, biosynthesis, structure determination and biological activities. J Antibiot. 2012;65:541-9.

25. Cramer B, Königs M, Humpf HU. Identification and in vitro cytotoxicity of ochratoxin A degradation products formed during coffee roasting. J Agric Food Chem. 2008;56:5673-81.

26. Rubert J, León N, Sáez C, Martins CP, Godula M, Yusà V, et al. Evaluation of mycotoxins and their metabolites in human breast 
milk using liquid chromatography coupled to high resolution mass spectrometry. Anal Chim Acta. 2014;820:39-46.

27. Waseem A, Shah AS, Sajjad A, Siddiqi AR, Nafees M. Human exposure to mycotoxins: a retrospective review of leading toxins and metabolites in human biological matrices. J Chem Soc Pak. 2014;36:1196-214.

28. Warth B, Sulyok M, Berthiller F, Schuhmacher R, Krska R. New insights into the human metabolism of the Fusarium mycotoxins deoxynivalenol and zearalenone. Toxicol Lett. 2013;220:88-94.

29. Mally A, Solfrizzo M, Degen GH. Biomonitoring of the mycotoxin Zearalenone: current state-of-the art and application to human exposure assessment. Arch Toxicol. 2016;90:1281-92.

30. Beyer M, Ferse I, Humpf H-U. Large-scale production of selected type A trichothecenes: the use of HT-2 toxin and T-2 triol as precursors for the synthesis of d 3-T-2 and d 3-HT-2 toxin. Mycotoxicol Res. 2009;25:41-52.

31. Hübner F, Harrer H, Fraske A, Kneifel S, Humpf H-U. Large scale purification of B-type fumonisins using centrifugal partition chromatography (CPC). Mycotoxicol Res. 2012;28:37-43.

32. Bretz M, Beyer M, Cramer B, Knecht A, Humpf H-U. Thermal degradation of the Fusarium mycotoxin deoxynivalenol. J Agric Food Chem. 2006;54:6445-51.

33. Cramer B, Harrer H, Nakamura K, Uemura D, Humpf H-U. Total synthesis and cytotoxicity evaluation of all ochratoxin A stereoisomers. Bioorg Med Chem. 2010;18:343-7.

34. Cramer B, Bretz M, Humpf H-U. Stable isotope dilution analysis of the fusarium mycotoxin zearalenone. J Agric Food Chem. 2007;55: 8353-8.

35. Bittner A, Cramer B, Humpf H-U. Matrix binding of ochratoxin A during roasting. J Agric Food Chem. 2013;61:12737-43.

36. Welsch T, Humpf H-U. HT-2 toxin 4-glucuronide as new T-2 toxin metabolite: enzymatic synthesis, analysis, and species specific formation of T-2 and HT-2 toxin glucuronides by rat, mouse, pig, and human liver microsomes. J Agric Food Chem. 2012;60:10170-8.

37. Hickert S, Bergmann M, Ersen S, Cramer B, Humpf H-U. Survey of Alternaria toxin contamination in food from the German market, using a rapid HPLC-MS/MS approach. Mycotoxin Res. 2015;32:7-18.

38. Lohrey L, Marschik S, Cramer B, Humpf H-U. Large-scale synthesis of isotopically labeled ${ }^{13} \mathrm{C}_{2}$-tenuazonic acid and development of a rapid HPLC-MS/MS method for the analysis of tenuazonic acid in tomato and pepper products. J Agric Food Chem. 2013;61:114-20.

39. Muñoz K, Blaszkewicz M, Campos V, Vega M, Degen GH. Exposure of infants to ochratoxin A with breast milk. Arch Toxicol. 2014;88:837-46.

40. Eriksen GS, Pettersson H, Lindberg JE. Absorption, metabolism and excretion of 3-acetyl don in pigs. Arch Anim Nutr. 2003;57: 335-45.

41. Dänicke S, Brosig B, Kahlert S, Panther P, Reinhardt N, Diesing AK, et al. The plasma clearance of the Fusarium toxin deoxynivalenol (DON) is decreased in endotoxemic pigs. J Food Chem Toxicol. 2012;50:4405-11.

42. Maul R, Warth B, Kant J-S, Schebb NH, Krska R, Koch M, et al. Investigation of the hepatic glucuronidation pattern of the Fusarium mycotoxin deoxynivalenol in various species. Chem Res Toxicol. 2012;25:2715-7.

43. Šarkanj B, Warth B, Uhlig S, Abia WA, Sulyok M, Klapec T, et al. Urinary analysis reveals high deoxynivalenol exposure in pregnant women from Croatia. Food Chem Toxicol. 2013;62:231-7.

44. Njumbe Ediage E, Di Diana Mavungu J, Song S, Wu A, van Peteghem C, de Saeger S. A direct assessment of mycotoxin biomarkers in human urine samples by liquid chromatography tandem mass spectrometry. Anal Chim Acta. 2012;741:58-69.

45. Solfrizzo M, Gambacorta L, Lattanzio VMT, Powers S, Visconti A Simultaneous LC-MS/MS determination of aflatoxin M1, ochratoxin A, deoxynivalenol, de-epoxydeoxynivalenol, $\alpha$ and $\beta$-zearalenols and fumonisin B1 in urine as a multi-biomarker method to assess exposure to mycotoxins. Anal Bioanal Chem. 2011;401:2831-41.

46. Vishwanath V, Sulyok M, Labuda R, Bicker W, Krska R. Simultaneous determination of 186 fungal and bacterial metabolites in indoor matrices by liquid chromatography/tandem mass spectrometry. Anal Bioanal Chem. 2009;395:1355-72.

47. Cizdziel JV. Determination of lead in blood by laser ablation ICPTOF-MS analysis of blood spotted and dried on filter paper: a feasibility study. Anal Bioanal Chem. 2007;388:603-11.

48. EFSA CONTAM Panel. Scientific Opinion on the risks to human and animal health related to the presence of beauvericin and enniatins in food and feed. EFSA J. 2014;12:3802. 